Théologiques

Théologiques

\title{
Justice globale et positionnement du sujet racialisé chez les militants chrétiens du Nord
}

\section{Gada Mahrouse}

Volume 18, numéro 1, 2010

Les Églises chrétiennes et la mouvance altermondialiste

URI : https://id.erudit.org/iderudit/1003524ar

DOI : https://doi.org/10.7202/1003524ar

Aller au sommaire du numéro

\section{Éditeur(s)}

Faculté de théologie et de sciences des religions, Université de Montréal

ISSN

1188-7109 (imprimé)

1492-1413 (numérique)

Découvrir la revue

Citer cet article

Mahrouse, G. (2010). Justice globale et positionnement du sujet racialisé chez les militants chrétiens du Nord. Théologiques, 18(1), 115-136.

https://doi.org/10.7202/1003524ar

\section{Résumé de l'article}

$\mathrm{Au}$ cours des dernières années, le mouvement altermondialiste et les Forums sociaux mondiaux (FSM) ont fait l'objet de critiques sérieuses. En effet, dès le deuxième FSM en 2002, certains commençaient à se demander si ces rencontres ne se prêtaient pas à la « colonisation de la résistance " (Mngxitama 2005). D’autres ont remarqué et commenté la prédominance des groupes du Nord aux différents forums. Mon objectif est d'étudier le positionnement racialisé des groupes confessionnels et de leurs membres habitant les pays du Nord. La méthode utilisée pour comprendre le processus de racialisation est en grande partie influencée par les méthodologies féministe, postcoloniale et antiraciste ; celles-ci cherchent à révéler les façons dont la connaissance est produite pour établir et maintenir des relations inégales, ainsi que pour construire des subjectivités au moyen de représentations et de modes de pensée racialisés. L'une des questions pratiques que mon analyse soulève est la suivante : les activistes des pays du Nord devraient-ils même participer aux mouvements mis en cause ici ? De plus, étant donné les inquiétudes croissantes exprimées par le mouvement altermondialiste et les gens qui participent aux FSM, j'ai tenu à soulever des questions qui nécessitent un examen beaucoup plus poussé ; j’ai aussi voulu faire ressortir le besoin de recherches universitaires plus soutenues et d'études approfondies sur les liens existant entre l’Église, la théologie et le mouvement altermondialiste.
Ce document est protégé par la loi sur le droit d'auteur. L'utilisation des services d'Érudit (y compris la reproduction) est assujettie à sa politique d'utilisation que vous pouvez consulter en ligne.

https://apropos.erudit.org/fr/usagers/politique-dutilisation/ 


\title{
Justice globale et positionnement du sujet racialisé chez les militants chrétiens du Nord"
}

\author{
Gada MAhrousE** \\ Institut Simone de Beauvoir \\ Université Concordia
}

\section{Introduction}

La participation des peuples du Nord aux mouvements de défense des droits de la personne et de systèmes économiques justes et équitables est souvent perçue comme un signe encourageant de changement ${ }^{1}$. Il n'y a qu'à voir l'enthousiasme et l'énergie suscités actuellement par le mouvement altermondialiste. Ce mouvement social prometteur, encore relativement jeune, est explicitement fondé sur la solidarisation des pays par-delà les frontières géographiques et les différences, afin de revendiquer collectivement la justice globale. Ses objectifs? Mettre en valeur la coopération et l'interaction mondiales, la justice environnementale, la justice économique, la défense des droits des travailleurs et travailleuses, ainsi que la protection des

* Texte traduit de l'anglais. L'auteur tient à remercier Karine Fortier pour son aide à la traduction.

* Gada Mahrouse est professeur associé à l'Institut Simone de Beauvoir de l'Université Concordia. Ses recherches portent sur l'accompagnement international. Elles utilisent les concepts du post-colonialisme et les théories sur la racialisation et le genre. Elles mettent en lumière la remise en question et la reproduction des relations de pouvoir propres à l'accompagnement international par des Occidentaux. Elle a récemment publié (2009): "Transnational Activism/ Humanitarianism as a Racialized 'Politics of Life': The Christian Peacemaker Team Kidnapping in Iraq", Citizenship Studies, 13/4, p. 311-331.

1. D'autres termes tels que «occidental/non occidental» sont aussi souvent utilisés pour distinguer, d'une part, les pays et communautés riches et privilégiés et, d'autre part, les pays et communautés «non occidentaux» marginalisés aux points de vue économique et politique. Je préfère employer l'expression «Nord/Sud» dans le présent article parce qu'elle décrit mieux les relations dont il s'agit dans le mouvement altermondialiste. 
cultures et des droits des peuples autochtones. D'ailleurs, l'idée de l'«altermondialisme» vient d'une devise désormais populaire, «Un autre monde est possible», formulée lors du premier Forum social mondial (ou FSM ${ }^{2}$ ). Avec le temps, les rencontres du FSM ont fini par symboliser le mouvement altermondialiste et, dans les faits, ont permis aux militants et militantes et aux groupes d'intérêts de se rassembler. Les Églises des pays du Nord, de concert avec les ONG et les syndicats, ont participé aux FSM et joué un rôle crucial dans l'essor de ce mouvement.

Or, ces dernières années, le mouvement altermondialiste et les FSM ont fait l'objet de critiques sérieuses. Comme l'ont souligné plusieurs commentateurs, si le premier FSM de Porto Alegre, en 2001, avait apporté une lueur d'espoir et une promesse de changement, pour beaucoup, cet espoir a été de courte durée (Melber 2007; Manji 2007). En effet, dès le deuxième FSM en 2002, certains commençaient à se demander si ces rencontres ne se prêtaient pas à la «colonisation de la résistance» (Mngxitama 20053). D'autres se demandaient également si les stratégies et les idées mises de l'avant par le mouvement visaient vraiment des changements fondamentaux dans les systèmes de pouvoirs. D'Souza (2004) donne en ce sens un exemple très pertinent, l'annulation de la dette du tiers-monde, idée que les Églises ont grandement appuyée et énergiquement défendue aux FSM. D'Souza souligne que cette idée découle fondamentalement des notions de pardon et de compassion, ce qui ne tient pas compte du fait que «ce qu'on appelle dette est en fait l'expropriation du tiers- monde et un moyen de continuer à s'approprier la terre, le travail et les ressources naturelles de cette région du globe» (2004). Elle fait aussi remarquer que la Banque mondiale a proposé d'éliminer une partie de la dette du tiers-monde à la condition que les pays acceptent de se restructurer pour se conformer aux exigences de la

2. Au nombre des termes utilisés aux FSM, on retrouve "altermondialisme » ainsi que "justice globale ", "anti-mondialisation ", "altermondialisation» et autres expressions, chacune d'entre elles reflétant une vision et des politiques spécifiques.

3. Dès 2002, des forums plus radicaux et anticonformistes ont été organisés parallèlement aux FSM afin de créer un espace qui avait été perdu à cause de l'ostentation des FSM et de toute l'attention médiatique dont ils étaient l'objet. Par exemple, en 2004, lors du forum parallèle "Mumbai Resistance ", les sources de financement du FSM ont suscité beaucoup de questions. Par exemple, certains ont soulevé le fait que la Fondation Ford, anciennement contrôlée par la CIA, faisait partie des principaux bailleurs de fonds, Mngxitama remarque que «l'une des accusations les plus graves était que le FSM n'est rien de plus qu'une soupape et un lieu de dissidence autorisé; il ne menace pas vraiment les intérêts du capital global» (2005). 
«mondialisation». Plus important encore, selon D'Souza, bien que l'annulation de la dette du tiers-monde apporte un certain répit, reste à voir si elle mettra fin à l'exploitation et à la destruction continues de la vie sociale et culturelle de nombreuses sociétés. Je n'aborde pas ce sujet dans le but d'amorcer un débat - il y a certainement bon nombre de questions complexes et divers points de vue sur l'annulation de la dette du tiers-monde dont cet article ne traitera pas. L'exemple précédent est plutôt présenté comme une critique pertinente des mouvements mentionnés et de leurs fondements chrétiens.

D'autres ont remarqué et commenté la prédominance des groupes du Nord aux différents forums. Selon Manji, le FSM de Nairobi n'était pas différent de tout autre événement de ce genre régi par les lois du marché néolibéral. Selon lui, «le Nord blanc, avec son esprit de clocher hégémonique, était surreprésenté» (2007). De même, Mngxitama (2005) a remarqué avec justesse qu'ironiquement, le FSM n'a pas fourni une tribune aux opinions et aux problèmes des Noirs, et ce, même au Brésil où vit la plus vaste population noire à l'extérieur de l'Afrique ${ }^{4}$. À la lumière de ces critiques, certains militants du Nord remettent en question leur rôle dans le mouvement et s'efforcent de militer en faveur de la justice sociale en adoptant des approches qui, paradoxalement, ne reproduiront pas les rapports de pouvoir déjà existants. Comme le thème de ce numéro de Théologiques l'indique, les théologiens, les universitaires et les membres de groupes confessionnels qui participent de diverses façons à l'altermondialisme se sont aussi engagés à examiner en profondeur les liens existant entre la théologie, la mondialisation et les rapports de forces actuels, ainsi que leurs propres rôles dans ce mouvement.

L'un des objectifs de ce numéro spécial est d'examiner l'implication des membres de groupes confessionnels dans certaines des nombreuses contradictions qui existent au sein du mouvement. Dans cette optique, j'ai décidé de commencer mon article par l'étude du positionnement racialisé

4. Quoique troublantes, ces critiques ne sont pas étonnantes. L'idée des pays du Nord solidaires des pays du Sud, bien que porteuse d'espoir, soulève des inquiétudes parce que l'histoire de différents régimes impérialistes nous a montré que de telles tentatives d' «aide» donnent souvent prétexte au néocolonialisme (voir Jean-Klein 2002). De plus, l'histoire des mouvements sociaux (y compris les mouvements à l'échelle nationale tels le mouvement féministe et le mouvement des droits civiques américains) nous a appris que, même avec les intentions les plus louables en termes de solidarité, les gens de couleur ne sont souvent pas traités comme des égaux dans de tels contextes (Roth 2005; Thompson 2001). 
des activistes du Nord qui militent à l'échelle mondiale. J'apporte donc ma modeste contribution en proposant des idées qui, je l'espère, encourageront la réflexion et la discussion chez ceux et celles qui savent par expérience comment sont racialisées les personnes engagées dans l'activisme mondial et les mouvements reliés aux Églises. Avant d'aborder la discussion, je tiens à souligner que je n'étudie pas la théologie; je veux aussi réfléchir à la tâche délicate que constitue la critique, par une non-chrétienne, des interventions liées aux Églises de même que la critique de l'altermondialisme par une observatrice externe. Cela dit, j'aimerais que les lecteurs sachent que je traiterai du sujet avec humilité et circonspection, de même qu'avec la conscience aiguë de mon manque de familiarité avec la longue histoire et avec le grand rayon d'action de l'activisme social relié aux Églises. J'éviterai donc les affirmations définitives et inviterai plutôt les lecteurs à faire eux-mêmes une analyse plus approfondie et plus nuancée du sujet. Je cherche particulièrement à donner des pistes de réflexion sur des points précis non abordés ici - points qui deviendraient évidents lors d'une étude plus contextualisée et historicisée des éléments suivants : distinctions entre les différents types d'Églises (p. ex. dénominations spécifiques, coalitions œcuméniques, réseaux interconfessionnels, etc.); différences entre les rôles joués par les membres des différents groupes ${ }^{5}$; diversité des actions et mouvements sociaux liés aux Églises (p. ex., visites de courte durée par opposition à la solidarité et à la collaboration à long terme); ainsi que différences dans le positionnement des participants (aux niveaux culturel, politique, économique, géographique, etc.).

Toutefois, l'inquiétude que je ressens en abordant le sujet est quelque peu allégée par ce que je considère comme une double approche du rôle de la foi et de la spiritualité dans les mouvements sociaux (voir Jones de Almeida 2007). D'une part, je respecte et peux comprendre que la foi et la spiritualité peuvent pousser les gens affiliés aux Églises à se dresser contre l'injustice. D'autre part, je suis d'accord avec ceux qui prétendent que présumer de l'innocence de telles activités est naïf et dangereux, surtout à la lumière du rôle de l'Église dans les injustices commises et perpétuées avec violence (Jones de Almeida 2007, 188).

Mon intérêt pour le présent sujet découle de mes recherches sur la question de la race dans les mouvements transnationaux de solidarité sociale. Je

5. Cette distinction est claire, par exemple, entre l'approche passive empruntée par le projet Canada-Guatemala pour apporter son soutien et l'approche plus «interventionniste» adoptée par les membres du CPT au Guatemala. 
m'intéresse particulièrement aux personnes du Nord qui se sont rendues dans divers lieux géopolitiques pour s'opposer à la violence militaire, à la guerre et à l'occupation, ou encore pour promouvoir des solutions pacifiques. Beaucoup d'entre elles faisaient partie d'organisations et de délégations reliées à l'Église (ou fondées sur la foi chrétienne ${ }^{6}$ ). Au nombre des groupes que j'ai étudiés et dont je discuterai ici figurent entre autres un programme d'accompagnement œcuménique ou de suivi établi par l'Église Unie du Canada, les Christian Peacemaker Teams et le Project Accompaniment. Aux fins de ma recherche, j'ai examiné certaines publications produites dans le cadre de ces projets de solidarité reliés aux Églises; j'ai aussi mené des entrevues approfondies avec des Canadiens anglophones blancs qui ont milité en faveur de la justice sociale dans divers endroits à l'étranger soit en tant que membres des groupes chrétiens mentionnés précédemment, soit à titre de chrétiens pratiquants poussés par leur foi à se joindre à des groupes laïques ${ }^{7}$.

Mon objectif est d'étudier le positionnement racialisé des groupes confessionnels et de leurs membres habitant les pays du Nord. La méthode utilisée pour comprendre le processus de racialisation est en grande partie influencée par les méthodologies féministe, postcoloniale et antiraciste; celles-ci permettent de révéler les façons dont la connaissance est produite pour établir et maintenir des relations inégales, ainsi que pour construire des subjectivités au moyen de représentations et de modes de pensée racialisés. Bien que les termes «antiraciste», «blancheur» et "postcolonial» soient principalement employés par les chercheurs universitaires anglophones, je les utilise ici parce que je considère qu'ils pourraient être utiles aux communautés et universitaires francophones voulant s'attaquer à ces questions ${ }^{8}$. Ceci dit, "race» signifie ici une identité fluide, construite et produite (Hall 1992; Dyer 1997). Par «racialisé», j'entends le "processus dialectique de la signification » par lequel des traits sont attribués à certains corps et états (Miles 1989, 7). À cela on peut ajouter l'idée que les processus de racialisation sont étroitement liés au genre et à la classe, et que le

6. Appelés communément «accompagnateurs", «témoins-observateurs» ou «boucliers humains », les activistes ont comme fonctions principales de protéger les personnes menacées de violence en attirant l'attention sur leur propre présence «internationale », d'effectuer une surveillance, ainsi que de documenter et de diffuser de l'information sur ce qu'ils ont observé.

7. Quinze entrevues ont été réalisées entre mars et mai 2005.

8. Pour plus de renseignements à ce sujet, voir Maillé (2007). 
discours racialisé ne s'exprime pas nécessairement en termes de «race» et peut inclure des pratiques et expressions discursives cachées derrière d'autres concepts, nommément la religion, aux fins du présent article (Goldberg 1993). En outre, en élaborant une théorie sur la relation entre la race et l'identité religieuse, Richard Dyer stipule que la blancheur est constituée à travers la chrétienté et l'impérialisme. Il prend toutefois bien soin de souligner qu'il n'affirme pas que la chrétienté est essentiellement blanche; il reconnaît en effet qu'elle est pleine de vitalité en Afrique, en Amérique du Sud et dans les églises noires d'Europe et d'Amérique du Sud (1997, 17). À son avis, néanmoins (même si l'observance des préceptes de la chrétienté décline peut-être), les modes de pensée et les sentiments prônés par la «chrétienté » caractérisent encore la culture et la conscience de l'Europe, ainsi que celles des colonies et anciennes colonies (notamment les États-Unis) à qui la chrétienté a donné le jour $(1997,15)^{9}$. Dyer ne suggère pas l'existence d'une «chrétienté» monolithique ou essentialiste, ou encore d'une «culture et d'une conscience européennes ${ }^{10}{ }^{»}$; il tente plutôt de saisir le processus signifiant selon lequel les pays du Nord sont considérés comme chrétiens et ceux du Sud comme non chrétiens, et de comprendre les relations de pouvoir qui en découlent.

Cet article est donc une étude préliminaire visant à présenter à ceux qui détiennent les privilèges (relatifs) inhérents au fait d'être des chrétiens du Nord, certaines questions importantes et pourtant souvent ignorées. Comme je le démontrerai, pour comprendre la dynamique racialisée derrière le pouvoir de ceux pour qui l'identité religieuse ou la foi a joué un rôle dans leur activisme, il faut se pencher sur la façon dont l'identité chrétienne, entre autres facteurs, fait de la blancheur un positionnement ou système dominant racialisé. Par exemple, au cours de mes lectures et de mes entrevues, j'en suis venue à constater que, dans la plupart des cas, il

9. En outre, comme dans toutes les catégories sociales, les limites de la blancheur changent dans le temps et l'espace. Par exemple, en étudiant les manières dont la blancheur juive est construite, Kaplan (1998) décrit la façon complexe dont les expériences de blancheur émergent de différentes histoires géographiques et sociales; elle indique de plus que les groupes identifiés comme blancs dans un certain contexte peuvent être racialisés différemment dans d'autres.

10. Comme l'un des critiques anonymes de mon article a eu l'obligeance de le remarquer, toute l'histoire de l'action sociale progressiste chrétienne témoigne du caractère diversifié et contesté de la "chrétienté » dans le Sud et le Nord. Pour plus de renseignements sur la diversité des chrétientés, voir Dale 2008; http://www.journalofworldchristianity.org/index. php/jowc. 
existe deux points de vue divergents sur les questions de race chez les personnes affiliées à des Églises qui contribuent à des projets en faveur de la justice sociale à l'étranger: il y a celles qui croient que leurs intentions de justice sociale et leur foi chrétienne les aident à transcender la race et à adopter un positionnement "neutre", et celles qui croient que les questions de positionnement racialisé doivent être constamment examinées et qui trouvent des façons de se réconcilier avec leur positionnement dominant, souvent en faisant appel aux concepts d'amitié et de collaboration. En me basant principalement sur les entrevues que j'ai menées, je soulèverai des questions sur ces deux points de vue. Dans la troisième partie, je résumerai mon analyse du cas très médiatisé de l'enlèvement d'un membre du groupe Christian Peacemaker Teams en Irak, pour affirmer que les rôles de ces activistes sont «surdéterminés" par la race de plusieurs façons. Je terminerai l'article en considérant certaines des implications de cette analyse pour les membres de groupes confessionnels engagés dans le mouvement altermondialiste.

\section{Intentions honorables et positionnement neutre d'un «tiers»}

En écrivant sur les Canadiennes spécialistes du développement en Afrique, Barbara Heron (2004) a expliqué que l'idée d' "intentionnalité » intervient toujours et éclipse les discussions sur la race, en ce sens que beaucoup invoquent des motifs et objectifs qu'ils jugent «honorables » pour justifier leurs actions à l'étranger. De même, dans plusieurs ouvrages écrits par des personnes ayant contribué à divers projets dirigés par l'Église à l'étranger, les références à la race sont quasi inexistantes, ou alors les différences racialisées entre les activistes et ceux qu'ils aident sont passées sous silence. Par exemple, le livre de Nepstad (2002) sur le projet Witness for Peace ${ }^{11}$ ou celui d'Anderson (2003), Weaving Relationship: Canada-Guatemala Solidarity, révèlent ceci: un grand nombre de ces activistes croient que leur foi les aide à transcender les différences de race, de classe, d'ethnie, de nation et d'idéologie, parce qu'ils estiment partager avec les populations qu'ils veulent aider une «identité collective » fondée sur la chrétienté progressiste. Comme certains l'ont souligné, les questions raciales sont rarement analysées dans les mouvements reliés aux Églises parce que l'on hésite

11. Witness for Peace est un groupe qui a amené plus de 4000 Nord-Américains à se joindre à des délégations se rendant dans les zones de guerre du Nicaragua pour constater de visu les effets de la guerre des Contras. 
à critiquer les efforts et les initiatives salués ailleurs comme honorables (Weber 2006).

En lien avec l'idée d'intentions honorables, on retrouve les orientations philosophiques et spirituelles non partisanes de nombreux groupes reliés aux Églises. Puisque les activistes sont encouragés à agir comme tiers et à ne pas être aveuglés par des idées partisanes, il serait intéressant de déterminer en détail si les participants à divers projets à l'étranger en viennent à se percevoir comme des acteurs impartiaux. Bien que je n'ai mené qu'un nombre limité d'entrevues, celles-ci indiquent clairement qu'il serait utile de réaliser plus d'études sur ce positionnement de «tiers» comme dynamique racialisée. De fait, mes recherches suggèrent que l'idée de «tiers » est particulièrement fréquente dans des endroits comme la Palestine et Israël, où le conflit est souvent décrit comme une lutte entre musulmans et Juifs. Selon une militante, dans ce contexte, les chrétiens ont une identité plus «neutre» pour des raisons évidentes. En effet, quelques-uns des activistes interrogés, qui sont allés en Palestine et en Israël dans le cadre du Programme œcuménique d'accompagnement en Palestine et Israël (EAPPI), ont déclaré qu'ils abordaient le conflit israélien avec compassion pour les «deux» parties, en vertu d'une impartialité de principe. Une militante explique que l'objectif est «d'essayer de se tenir au milieu ». Elle poursuit ainsi :

Nous soutenons les gens qui souffrent. [...] Le programme est fondé sur la théologie de la libération, qui est l'appel de Dieu nous invitant à prendre soin des pauvres et des opprimés. Selon la théologie de la libération, ces derniers occupent une place spéciale dans le cœur de Dieu. Notre objectif est donc la libération des opprimés, la liberté pour tous. Nous militons en faveur de la liberté pour tous. [...] Je suppose, en fait, que nous défendons les droits de la personne et, qu'en d'autres mots, nous nous battons pour la justice, la paix et la sécurité de tous ${ }^{12}$.

Cette réponse était typique. Le recours à la notion de neutralité était une réponse courante aux questions liées à la race et au positionnement dominant des activistes. En outre, pour certains, l'idée de neutralité reposait non seulement sur leur identité chrétienne, mais elle était renforcée par leur identité canadienne. Ceci ressortait particulièrement dans le discours d'une activiste qui déclarait qu'en tant que Canadienne et chrétienne, elle possédait la capacité d'être motivée par un désir d'aider pur et impartial. Tout en

12. Ceci est une des dix-huit entrevues en profondeur conduites auprès d'artivistes entre mars et mai 2005 lors d'un projet de recherche précédent. 
reconnaissant que le Canada était lui-même confronté à des problèmes de racisme, elle croyait que le fait d'être Canadienne lui conférait des compétences et des connaissances spéciales en matière de multiculturalisme.

Il est utile de faire ressortir plusieurs points à ce sujet. Premièrement, par leur discours sur la neutralité, certains activistes se présentent comme des sujets "non étiquetés ", rétablissant ainsi le pouvoir et l'invisibilité de leur blancheur (Dyer 1997). Deuxièmement, en se définissant comme un "tiers » dépolitisé cherchant à "apporter la liberté à tous ", ils impliquent même par inadvertance qu'ils sont menés par leur altruisme, alors que les activistes locaux des zones où ils se rendent sont, quant à eux, motivés par leur intérêt personnel. Autrement dit, en se positionnant en faveur des droits de la personne, de la justice et de la paix, on présume que les gens enlisés dans un conflit n'aspirent pas à atteindre ces mêmes buts. De plus, le fait d'amalgamer chrétienté et neutralité a pour effet d'effacer les exclusions racistes faites au nom de la chrétienté.

Ayant remarqué que plusieurs adoptent l'optique de la transcendance de la race ou encore de la neutralité pour éviter de s'interroger sur leur positionnement racialisé, je désire également examiner le point de vue de ceux qui étaient troublés par les questions de la race et du pouvoir. En fait, je crois que c'est dans les efforts conscients pour être antiraciste que l'emprise hégémonique des relations de pouvoir racialisées est la plus évidente. Je traiterai maintenant de la perspective de ceux chez qui la conscience de la race est présente.

\section{Collaboration et amitié: être conscient de sa position}

Bien sûr, tous les groupes confessionnels n'ignorent pas les questions raciales; même ceux qui aspirent à un positionnement de tiers neutre ne se positionnent pas sans difficulté comme étant purement charitables ou altruistes. En fait, la plupart des gens interviewés se montraient, dans leur activisme et leurs actions de solidarité, résolument conscients de leurs privilèges et de leur citoyenneté racialisés. J'ai d'ailleurs été frappée par le fait que la plupart d'entre eux comprenaient leur positionnement dominant et ressentaient aussi beaucoup d'ambivalence à ce sujet. Dans sa critique du projet Witness for Peace, Clare Weber (2006) a aussi noté que certains membres de groupes confessionnels sont conscients qu'en plus de leur conférer un poids politique, le privilège d'être blanc influe de façon plus générale sur leurs projets. Son travail démontre clairement deux choses: d'une part, qu'une telle conscience du problème existe et, d'autre part, qu'il 
faut examiner les questions relatives au positionnement racialisé des activistes.

Dans ma recherche de matériel publié par des groupes confessionnels engagés dans des projets à l'étranger, j'ai découvert que la majorité de ces groupes avaient rédigé une déclaration officielle à propos des questions de privilège et de déséquilibre des pouvoirs. Bien que formulées de différentes façons, ces déclarations communiquent généralement le message suivant: le groupe considère qu'il agit en solidarité avec et non pas pour les gens qu'il aide et souligne que, par principe, il s'efforce de collaborer avec ceuxci. La déclaration du réseau Maritimes-Guatemala Breaking the Silence Network, affilié à l'Église Unie du Canada, en est un exemple typique:

Nous reconnaissons que les structures de l'oppression dans les pays du Sud sont enracinées dans les structures de privilège des pays du Nord, et pour amener du changement au Guatemala, nous devons aussi travailler en vue d'apporter du changement au Canada. Nous sommes unis à nos partenaires et aux autres Guatémaltèques œuvrant pour la justice sociale dans une lutte commune $^{13}$.

Avec leurs déclarations officielles, ces groupes semblent mettre en pratique ce que la théorie féministe appelle la «politique de la différence ou de la position ${ }^{14}$. Ainsi, la majorité semble reconnaître que même si la solidarité peut être une stratégie efficace et importante, elle comporte implicitement le grand danger de réattribuer le pouvoir aux membres des groupes dominants, en raison d'inégalités inhérentes. Nul ne peut contester l'idée que la compréhension et l'application de la notion de différences de pouvoir dans les principes directeurs des mouvements affiliés aux Églises constituent une première étape nécessaire et importante. Toutefois, je ne présumerai pas simplement qu'il s'agit d'un fait accompli. En m'inspirant principalement du travail des féministes antiracistes et postcoloniales, je démontrerai maintenant qu'il serait bon de scruter davantage la conscience des activistes et les déclarations officielles pour révéler certaines des dimensions racialisées cachées de leur position.

13. On peut retrouver cet extrait à l'adresse suivante: http://tatacentre.ca/partnerships/bts.

14. "Politique de la position" est une expression d'abord créée par A. Rich, dans les années 1980, pour examiner les relations de pouvoir au cours de la deuxième vague du mouvement féministe nord-américain. Elle met en doute l'idée que les femmes peuvent s'unir au-delà des frontières nationales et culturelles (voir Kaplan 1994). 
Effectivement, certaines critiques féministes antiracistes et postcoloniales nous mettent en garde contre les propos sur la conscience antiraciste et sur la politique de la position; elles démontrent en outre que certains modes de confession antiraciste sont rédempteurs et susceptibles non seulement de dé-historiciser les diverses histoires racistes, mais aussi de valoriser les auteurs de ces confessions (Roman 1997; Kaplan 1994; Ahmed 2004): en d'autres mots, le privilège blanc peut se répéter dès qu'on l'évoque, car le fait de l'admettre peut servir de preuve indiscutable d'un engagement antiraciste. Selon Heron, ces déclarations concrétisent les systèmes dont on reconnaît l'existence parce qu'elles sont susceptibles de laisser ceux qui nomment le privilège racial dans une "position doublement confortable: premièrement, ils démontrent qu'ils sont conscients de leur situation et critiques à son égard; deuxièmement, ils n'ont pas besoin d'agir pour détruire leur privilège » $(2005,344)$. Ces théoriciennes font de plus remarquer que le fait de reconnaître un privilège n'entraîne aucun changement concret. C'est pourquoi j'ai voulu savoir comment les activistes vont au-delà de leurs déclarations sur le fait d'être conscients de leurs privilèges et tentent d'aplanir les différences de pouvoir.

Point fort intéressant, pour s'accommoder de leur pouvoir, le minimiser et l'accepter, les activistes ont parlé à maintes reprises, au cours des entrevues, des notions d'amitié et de collaboration. Ils répétaient souvent qu'ils partaient à l'étranger pour travailler en collaboration et apprendre. Ils affirmaient ainsi que leur travail ne constituait pas une répétition du modèle colonial ou impérial qui était d'aller enseigner ou prêcher (comme dans le travail missionnaire). Par exemple, un membre du CPT a déclaré que son équipe veillait à ne pas présumer que ses membres savaient mieux que quiconque comment agir. Elle a ajouté qu'une partie de leur orientation philosophique les amenait à faire preuve d'autoréflexion et à constamment remettre en question leur rôle et leurs interventions. Elle a expliqué qu'elle se considérait comme une élève plutôt que comme «une experte». J'ai retrouvé un sentiment semblable chez une femme ayant pris part au projet de solidarité Canada-Guatemala. Selon elle, le mandat des membres de son groupe est d'offrir un soutien passif à titre d'observateurs plutôt que d'agir à titre de conseillers. Quand je leur ai demandé s'ils remettaient parfois en cause leur "présence là-bas ", plusieurs activistes ont insisté sur le fait qu'il était important pour eux que les groupes locaux aient souhaité leur présence. Pour beaucoup d'entre eux, le fait d'avoir été invité diminuait l'inquiétude ressentie face à leur «droit» d'être là. En outre, savoir qu'on désirait leur présence et qu'on avait besoin d'eux les réconfortait grandement. 
Pour comprendre comment ces points de vue et déclarations reproduisent le pouvoir racialisé de manière subtile, il est utile de se référer au questionnement critique de Sara Ahmed sur les tentatives des féministes de travailler à partir de la prémisse de l'amitié comme moyen de transcender le pouvoir. Le danger d'un tel fondement épistémique, selon Ahmed, est qu'il permet de cacher des divisions dans les relations de pouvoir et les relations matérielles $(2000,65)$. À partir de cette analyse, on peut conclure qu'en se percevant comme une présence désirée, nécessaire et aimée, les activistes sont libres de s'enorgueillir de leur participation à diverses actions mondiales. Ahmed qualifie de telles manœuvres de «fantasme postmoderne». Elles permettent aux Blancs ou Occidentaux de recevoir des félicitations pour avoir renoncé à leur autorité, comme leur habileté à bien écouter le démontre $(2000,64)$. Elle signale également que ce sont de tels efforts pour positionner l'Autre comme un égal qui peuvent masquer les façons dont les Blancs contribuent à l'oppression. En somme, il faut être conscient des limites du discours sur l'"amitié». Certaines pratiques précises mériteraient d'être examinées de plus près. Il importe également d'approfondir la recherche pour savoir si les organisations et les réseaux accordent plus d'attention à ces questions que les individus, et ce, dans quelle mesure.

\section{Surdéterminé par la race}

L'enlèvement sensationnel et largement médiatisé de James Loney, militant canadien-anglais de race blanche qui a accompagné le groupe Christian Peacemaker Teams en 2005 pour protester contre la guerre en Irak ${ }^{15}$, est un cas fort intéressant. Il permet en effet d'étudier le positionnement racialisé des activistes chrétiens participant à des actions de solidarité mondiale. Loney est l'un des quatre hommes qui ont été enlevés et secourus par la suite. L'image de Loney dans les médias canadiens que j'ai analysée en pro-

15. Quatre activistes anti-guerre voyageant avec le groupe Christian Peacemaker Teams (CPT) ont été kidnappés à Bagdad. Deux d'entre eux ont été identifiés comme étant des citoyens canadiens; le troisième était Américain et le quatrième, Britannique. En mars 2006, après quatre mois de captivité environ, une opération militaire composée de soldats britanniques, américains, canadiens et irakiens a permis de libérer trois des hommes. Ces derniers ont pu regagner leur pays de résidence. Peu de temps après l'annonce de l'enlèvement dans les médias, le public apprenait que le CPT est une organisation bien établie depuis longtemps, dont les membres sont guidés par la foi chrétienne et le principe de la non-violence. Le CPT assure une présence continue en Irak depuis octobre 2002, soit le début de la plus récente invasion militaire américaine. 
fondeur dans une autre publication (voir Mahrouse 2009), nous éclaire en particulier de manière convaincante sur les façons dont l'activisme de solidarité peut être limité par le positionnement racialisé. Afin de soulever de nouvelles questions sur la dynamique racialisée de l'activisme international inspiré de la foi chrétienne, il serait utile de résumer brièvement certains points significatifs de mon analyse antérieure.

Par exemple, en élargissant la discussion au-delà des intentions des activistes du CPT et de leurs multiples actions couronnées de succès, je me suis plutôt attachée à étudier la couverture médiatique de l'histoire du CPT en Irak. J'ai démontré que, malgré les admirables intentions de Loney et ses efforts inlassables pour s'opposer au discours dichotomique dominant du bon chrétien/méchant musulman qui était courant au moment de l'enlèvement, les médias grand public ont quand même réussi à le présenter comme un "héros et guerrier chrétien blanc ", image subordonnée à sa contrepartie: «le musulman terroriste ${ }^{16}$ ». Loney a tenté d'expliquer la violence en Irak comme une conséquence du militarisme et de l'exploitation impérialiste. Il a aussi essayé de décrire les kidnappeurs comme des gens désespérés poussés à utiliser cette forme de violence. Malgré cela, ce qui ressortait de la couverture médiatique était la plupart du temps conforme à la version contemporaine du paradigme orientaliste du «choc des civilisations " (Saïd 1978). Comme certains l'ont observé, depuis le 11 septembre, la chrétienté fait partie intégrante des constructions de l'identité blanche (Ross 2005). Ces auteurs expliquent ainsi comment les réactions aux attaques ont servi à renforcer l'idée d'une Amérique du Nord chrétienne. Ross fait remarquer, par exemple, que les politiciens terminent leurs discours par l'invocation obligatoire "Que Dieu bénisse l'Amérique ». Pour lui, cette forme de «patriotisme» politico-religieux signifie que la dissidence n'a pas sa place. Il ne faut laisser aucune possibilité de douter que l'Amérique est entièrement blanche et chrétienne, et qu'elle est engagée dans une croisade contemporaine $(2005,240)$. Dans ce climat sociopolitique extrêmement tendu et rempli de dissension, j'ai avancé l'idée que même si Loney s'est rendu en Irak pour s'opposer à la politique étrangère de pays nord-américains tels les États-Unis et le Canada, il a fini par représenter, par inadvertance, l'amalgame de la blancheur hégémonique et de la chrétienté qu'on retrouve dans ces pays.

16. Je fais principalement référence à l'entrevue en profondeur faite avec Loney à l'émission de radio de la CBC intitulée The Current, le 6 juin 2006. 
J'ai soutenu qu'il était important de remarquer les paramètres selon lesquels l'histoire de son enlèvement pouvait être racontée et comment ces paramètres sont déjà fixés par la compréhension actuelle, racialisée du soi et de l'Autre (Saïd 1978). En d'autres mots, les médias semblaient ignorer son désaccord ou avaient décidé de ne pas en tenir compte et ont utilisé son histoire pour leurs propres fins racialisées. Par exemple, certains médias gais et lesbiens du Canada ont décrit l'enlèvement de Loney avec un parti pris qui révèle clairement l'emprise tenace des discours orientalistes. L'homosexualité de Loney avait été dissimulée au public jusqu'à son retour au pays, de peur de compromettre encore plus sa sécurité, ce qui est compréhensible. Il est très intéressant de constater que cette décision de dissimuler l'orientation sexuelle de Loney a permis à une partie de la communauté gaie et lesbienne de se constituer comme groupe civilisé face à la barbarie homophobe qui caractérisait l'Irak (Gulliver 2006; Marchildon 2006). Paradoxalement, comme Loney l'a répété lui-même à plusieurs reprises, sa «sortie du placard» au Canada a eu certaines répercussions. Par exemple, Loney et ses supporteurs allèguent que la publicité entourant sa sexualité a entraîné la fermeture d'un camp catholique ontarien où il avait été employé (CBC 2006). Un an plus tard, une invitation à présenter une allocution dans le cadre d'une conférence catholique romaine lui a été retirée en raison de l'opposition de l'Église à l'homosexualité (CBC 2007). Dans ces circonstances, le CPT et Loney se sont continuellement efforcés de contester l'idée raciste selon laquelle l'homophobie n'existe que «là-bas ». Loney a déclaré publiquement qu'il y avait un "parallèle» entre certaines des expériences vécues au Canada et les motifs qui l'avaient poussé à dissimuler son orientation sexuelle en Irak. Il a ajouté que la règle du silence rencontrée ici par moments «ressemblait beaucoup à celles auxquelles il était confronté en Irak» (CBC 2007). Cependant, en dépit de ses efforts résolus et habiles pour s'opposer au discours dominant, l'image médiatique de Loney a largement servi à accentuer et à normaliser le paradigme racialisé actuel selon lequel les habitants des pays du Nord (surtout les Canadiens) sont des êtres civilisés et pacifiques.

En attirant l'attention sur ces contradictions, mon but n'est pas de sous-entendre que les activistes comme Loney ne devraient pas s'engager dans des actions de solidarité, ni de suggérer que les interventions de celuici ont été inutiles. L'attention médiatique portée à l'enlèvement de Loney a pu servir à miner le privilège racialisé en réorientant cette attention sur les personnes toujours à risque d'être enlevées ou toujours en prison, autant en 
Irak qu'au Canada: c'est l'un des succès remarquables du CPT ${ }^{17}$. Mon analyse des modes de représentation de l'enlèvement de Loney fait simplement ressortir la nécessité de mettre en évidence les paradoxes troublants qui émergent avec l'utilisation du privilège occidental blanc, et d'y réagir. En m'inspirant de l'idée de l'homme noir "surdéterminé » par sa race, telle que posée par Fanon (1992), et en l'inversant, je constate qu'une dynamique semblable se répète avec le concept de la blancheur: étant donné ce que son corps représente, les façons dont Loney a été vu (et entendu) parachèvent et confirment la compréhension que nous avons de nous et d'eux. Autrement dit, il est facile de voir que parce que l'activisme est enraciné et formulé dans des tropes impérialistes, même les activistes les plus critiques, les plus dévoués et les plus familiarisés avec les pratiques médiatiques peuvent servir involontairement et sans le savoir à renforcer les stéréotypes courants. J'insiste sur le fait que mon but n'est ni de critiquer Loney, ni d'ignorer les nombreuses personnes que les efforts anti-guerre de Loney et du CPT ont peut-être interpellées et émues. Je ne veux que faire la distinction entre la forte emprise du paradigme racialisé actuel et la voix dissidente de Loney, "plus faible ${ }^{18}$. Je veux de plus soulever certaines des questions éthiques plus vastes ayant trait aux pratiques de solidarité dans le cadre des relations de pouvoir asymétriques, à l'échelle mondiale.

\section{Pistes de conclusion}

Il ressort de mon analyse qu'il faut remettre en cause de nombreux éléments du phénomène de l'aide étrangère par des Occidentaux. En effet, l'idée de "prêter» ou d'utiliser un privilège sous la forme d'une présence racialisée concrète, bien que simple et de bonne foi, à première vue, peut avoir des effets très contradictoires. Ce genre d'activisme est toujours une tentative risquée et équivoque; malgré cela, je me suis surtout attardée aux discours d'intentionnalité et de neutralité pour étudier comment ceux-ci peuvent permettre à certains activistes chrétiens d'éviter les questions de race en lien avec leur positionnement dominant de citoyens du Nord. En

17. Une dynamique tout aussi fascinante est apparue après l'enlèvement de Loney. Elle se rattache à la manifestation publique de solidarité de la part de membres des communautés musulmanes, à l'égard du CPT. Pour une discussion plus approfondie de ces événements et de cette dynamique, voir Mahrouse (2009).

18. Je suis reconnaissante à l'un de mes critiques anonymes pour ses commentaires sur ce point. 
voulant remettre en question ce que Shohat qualifie de «métarécits de libération ", j'ai tenté de contester la formule simpliste selon laquelle il ne faut pas voir une forme de pouvoir transcendant ou hégémonique dans les solidarités $(1998,10)$. Mon analyse suggère en outre que le principe chrétien d'égalité, qui permet aux activistes de se voir comme un parti neutre, doit certainement faire l'objet d'un plus ample examen critique. Il pourrait être bénéfique pour les personnes engagées à l'étranger dans des actions de solidarité fondées sur la foi chrétienne d'envisager le fait que la notion de neutralité est remplie d'implications raciales; elle ne peut donc être utilisée que par les gens racialisés comme Blancs. Je me suis en outre inspirée de Mohanty pour suggérer que les activistes doivent sérieusement réfléchir à leur «localisation » psychique de même qu'à leur localisation géographique, historique et culturelle (2003, 106, je souligne). Pour déterminer l'effet d'ensemble de leur activisme, il importe de se pencher sur la façon dont ces rencontres les amènent à se percevoir.

Je tiens à insister sur le fait que la majorité des membres de groupes confessionnels du Nord participant à diverses activités de justice dans le monde entier, semblent très conscients des manières dont ils sont investis des privilèges racialisés de la blancheur et de leur citoyenneté occidentale. Mes conclusions révèlent toutefois que ceux qui sont conscients et inquiets de leur positionnement dominant peuvent parfois s'en accommoder en invoquant des notions d'amitié et de collaboration. Cela indique, entre autres, qu'il ne suffit pas d'admettre qu'il y a des différences de pouvoir, il faut aussi reconnaître que des notions d'amitié et de confiance n'éliminent pas les divisions racialisées du pouvoir (Ahmed 2000). Ainsi, même quand certains groupes et individus expriment des préoccupations anticoloniales et antiracistes, ils peuvent ignorer, dans leurs discours (souvent formulés en termes d'une revendication pour l'universalité des droits de la personne à l'échelle internationale), l'histoire, la contingence et le contexte (Grewal 1998, 518). Enfin, la section portant sur la couverture médiatique de l'enlèvement d'un membre du CPT démontre clairement que le corps des activistes a une signification racialisée. Je suggère de plus qu'il faut s'intéresser au fonctionnement insidieux du racisme et du pouvoir racialisé.

Pour en revenir aux critiques du FSM et du mouvement altermondialiste présentées au début de cet article, quelles conclusions précises peut-on tirer de cette discussion? Je n'ai pas traité ici de la mesure dans laquelle ces questions ont jusqu'à maintenant eu une influence sur certaines organisations et certains mouvements participants, ainsi que sur les processus du FSM. Je crois cependant que la discussion offre d'importantes considéra- 
tions générales aux activistes affiliés à des Églises et engagés dans des projets en faveur de la justice mondiale. D'abord, ils doivent reconnaître que, malgré leurs objectifs déclarés de justice globale et de changement social, certains mouvements et projets mondiaux normalisent et masquent en fait les hiérarchies. Ensuite, en adoptant une position vertueuse de neutralité ou en se concentrant sur leur intentionnalité, les activistes réaffirment la blancheur: ils rétablissent plutôt que contestent les relations actuelles puisque leur discours fait abstraction d'une complicité entre chrétiens et Canadiens dans la souffrance mondiale. Je suggère en outre qu'un processus de désapprentissage pourrait être l'une des façons de transcender les barrières conceptuelles, en amenant à remplacer les notions de subjectivité libérale par le concept de sujet historique. Je veux également attirer l'attention sur la nécessité de saisir toutes les complexités de la dynamique du pouvoir racialisé sous des angles qui permettent de reconnaître la potentialité, les contradictions et les actions impérialistes des activistes affiliés à des Églises. Il est alors possible de commencer à observer l'interaction entre la domination et l'assujettissement dans des contextes historiques et contemporains.

L'une des questions pratiques que mon analyse soulève est la suivante: les activistes des pays du Nord devraient-ils même participer aux mouvements mis en cause ici? Parfois, lorsque des analyses critiques exposent les façons insidieuses dont le racisme s'infiltre dans les interventions les plus bienveillantes, les gens tendent à penser qu'il serait préférable que les personnes occupant une position dominante ne participent pas à des activités militantes. Devons-nous relever le défi ? Étant donné que les citoyens des pays du Nord ont de la mobilité, des ressources et de l'autorité, devraientils s'engager? En réaction à la surreprésentation des gens du Nord aux FSM, par exemple, certains ont commencé à remettre en question leur présence (Melber 2007). Il s'avère essentiel d'étudier cette question en profondeur.

Le but du présent article n'est évidemment pas de décourager les efforts de justice sociale. En insistant sur le fait que des interventions en ce sens doivent continuellement être examinées de façon critique, j'ai tenté de mettre en lumière les dangers et les limites de certaines stratégies des activistes et, en outre, de faire connaître certains rouages moins apparents du pouvoir. C'est dans cet esprit, je crois, qu'il importe de se demander si, dans certaines circonstances, la contribution des gens du Nord ne serait pas plus efficace s'ils n'assistaient pas aux activités organisées dans les pays du Sud. Les questions mises en évidence ici permettent de repenser l'action de solidarité 
mondiale pour éviter plus facilement la dynamique de pouvoir racialisé. À cette fin, ma réflexion encourage les mouvements sociaux mondiaux à se concentrer sur les inégalités de niveau de vie entre les différentes nations, l'inégalité d'accès aux ressources économiques, l'héritage du colonialisme et le commerce international. Les effets de la mondialisation et de l'impérialisme occidental sont tels qu'il est essentiel que nous prêtions attention au fait que certaines interventions ne peuvent se situer en dehors des plus grands systèmes de pouvoirs hégémoniques occidentaux. Compte tenu de cet objectif, j'espère que mon article favorisera l'examen de pratiques transformatrices. De plus, étant donné les inquiétudes croissantes exprimées par le mouvement altermondialiste et les gens qui participent aux FSM, j'ai tenu à soulever des questions qui nécessitent un examen beaucoup plus poussé. J'ai aussi voulu faire ressortir le besoin de recherches universitaires plus soutenues et d'études approfondies sur les liens existant entre l'Église, la théologie et le mouvement altermondialiste.

\section{Références}

Ahmed, S. (2000), Strange Encounter: Embodied Others in Post-coloniality, Londres/New York, Routledge.

(2004), «Declarations of Whiteness: The Non-performativity of Anti-racism ", Borderlands Ejournal, 3, p. 1-15.

ANDERSON, K. (2003), Weaving Relationships: Canada-Guatemala Solidarity, Waterloo, Wilfrid Laurier University Press.

Burman, T. (2006), "Thanks, Mr. Loney. Just Don't Read the News », <http://www.cbc.ca/news/about/burman/20060327.html>, consulté le 6 juin 2008.

CBC (2006), «Loney Says Camp Closing Because of His Homosexuality ", <http://www.cbc.ca/canada/story/2006/06/20/loney-camp.html>, consulté le 3 novembre 2006.

(2007), «Catholic Conference Retracts Invitation to Former Iraq Hostage ", <http://www.cbc.ca/canada/manitoba/story/2007/10/26/ loney-winnipeg.html>, consulté le 6 juin 2008.

DALE, I. (2008), «World Christianity. An Introduction », Journal of World Christianity, 1, p. 1-26.

D'souZA, R. (2004), "The World Social Forum Revisited: Back to Basics?", XCP: Cross Cultural Poetics, 14, p. 42-45. 
DYER, R. (1997), "The Matter of Whiteness », White, p. 1-40.

Fanon, F. (1992), "The Fact of Blackness », dans J. Donald et A. Rattansi, dir., Race, Culture and Difference, Londres, The Open University, p. 220240 .

Goldberg, D.T. (1993), Racist Culture: Philosophy and the Politics of Meaning, Cambridge, Blackwell.

Grewal, I. (1998), "On the New Global Feminism and the Family of Nations: Dilemmas of Transnational Feminist Practice », dans E. Shohat, dir., Talking Visions: Multicultural Feminisms in a Transnational Age, Cambridge, The MIT Press, p. 501-530.

Gulliver, T. (2006), "A Life-or-Death Closet: In Person, Christian Peacekeeper's Partner Back in the Picture », <http://www.xtra.ca/public/ Toronto/A_lifeordeath_closet-1748.aspx>, consulté le 18 juin 2009.

Hall, S. (1992), "New Ethnicities ", dans J. Donald et A. Rattansi, dir., Race, Culture and Difference, Londres, The Open University, p. 252-259.

Heron, B. (2004), "Gender and Exceptionality in North-South Interventions: Reflecting on Relations ", Journal of Gender Studies, 13, p. 117-127.

(2005), "Self-Reflection in Critical Social Work Practice: Subjectivity and the Possibilities of Resistance ", Reflective Practice, 6, p. 341-351.

Jean-Klein, I. E. (2002), "Alternative Modernities, or Accountable Modernities? The Palestinian Movement(s) and Political (Audit) Tourism During the First Intifada ", Journal of Mediterranean Studies, 12, p. 43-79.

Jones DE ALmeIDA, A. F. (2007), «Radical Social Change: Searching for a New Foundation / Incite! Women of Color Against Violence ", dans The Revolution Will not Be Funded: Beyond the Non-Profit Industrial Complex, Cambridge, South End Press, p. 185-195.

Kaplan, C. (1994), "The Politics of Location as Transnational Feminist Practice ", dans I. Grewal et C. Kaplan, dir., Scattered Hegemonies: Postmodernity and Transnational Feminist Practices, Minneapolis, University of Minnesota Press, p. 137-152.

(1998), «Beyond the Pale: Rearticulating U.S. Jewish Whiteness », dans E. Shohat, dir., Talking Visions: Multicultural Feminisms in a Transnational Age, Cambridge, The MIT Press, p. 451-484. 
Mahrouse, G. (2009), «Transnational Activists, News Media Representations, and Racialized 'Politics of Life': The Christian Peacemaker Team Kidnapping ", Iraq Citizenship Studies, 13, p. 311-331.

MAILlÉ, C. (2007), «Réception de la théorie postcoloniale dans le féminisme québécois ", Recherches féministes, 20, p. 91-111.

ManjI, F. ( 2007), «World Social Forum : Just Another NGO Fair?», <http://www.pambazuka.org/en/category/features/39464>.

MARChiLdon, G. (2006), «When Silence is Golden », <http://www.egale.ca/ index.asp ?lang=E\&menu=1\&item=1314>, consulté le 30 Avril 2007.

Melber, H. (2007), "Is Another World Possible ? Some (Self-) Critical Reflections on the World Social Forum in Nairobi 2007-02-01 ", <http://pambazuka.org/en/category/comment/39607>.

MiLEs, R. (1989), Racism, New York, Routledge.

Mngxitama, A. (2005), "WSF: The Colonisation of Resistance? Network Institute for Global Democratization ", <http://www.nigd.org/docs/ nigd/dos/WSFAndileMngxitama>, consulté en août 2010.

Mohanty, C. T. (2003), Feminism Without Borders: Decolonizing Theory, Practicing Solidarity, Durham, Duke University Press.

NePSTAD, S. E. (2002), "Creating Transnational Solidarity: The Use of Narrative in the US Central America Peace Movement ", dans J.G. Smith et H. Johnston, dir., Globalization and Resistance: Transnational Dimensions of Social Movements, Lanham, Rowman \& Littlefield, p. 133-149.

Roman, L. (1997), "Denying (White) Racial Privilege: Redemption Discourses and the Uses of Fantasy ", dans M. Fine, L. Weis, L. C. Powell et L. M. Wong, dir., Off White: Readings on Race, Power, and Society, New York, Routledge, p. 270-282.

Ross, T. (2005), «Whiteness After 9/11 ", Journal of Law and Policy, 18, p. 223-243.

Roth, B. (2004), Separate Roads to Feminism: Black, Chicana, and White Feminist Movements in America's Second Wave, Cambridge, Cambridge University Press.

SAID, E. (1978), Orientalism, Londres, Routledge.

Shонат, E. (1998), «Introduction", dans E. Shohat, dir., Talking Visions: Multicultural Feminism in Transnational Age, New York, MIT Press, p. 1-63. 
Thompson, B. (2001), A Promise and a Way of Life, Minneapolis/Londres, University of Minnestota Press.

Weber, C. (2006), Visions of Solidarity: U.S. Peace Activists in Nicaragua from War to Women's Activism and Globalization, New York, Lexington Books.

\section{Résumé}

Au cours des dernières années, le mouvement altermondialiste et les Forums sociaux mondiaux (FSM) ont fait l'objet de critiques sérieuses. En effet, dès le deuxième FSM en 2002, certains commençaient à se demander si ces rencontres ne se prêtaient pas à la «colonisation de la résistance» (Mngxitama 2005). D'autres ont remarqué et commenté la prédominance des groupes du Nord aux différents forums. Mon objectif est d'étudier le positionnement racialisé des groupes confessionnels et de leurs membres habitant les pays du Nord. La méthode utilisée pour comprendre le processus de racialisation est en grande partie influencée par les méthodologies féministe, postcoloniale et antiraciste; celles-ci cherchent à révéler les façons dont la connaissance est produite pour établir et maintenir des relations inégales, ainsi que pour construire des subjectivités au moyen de représentations et de modes de pensée racialisés. L'une des questions pratiques que mon analyse soulève est la suivante: les activistes des pays du Nord devraient-ils même participer aux mouvements mis en cause ici? De plus, étant donné les inquiétudes croissantes exprimées par le mouvement altermondialiste et les gens qui participent aux FSM, j'ai tenu à soulever des questions qui nécessitent un examen beaucoup plus poussé; j'ai aussi voulu faire ressortir le besoin de recherches universitaires plus soutenues et d'études approfondies sur les liens existant entre l'Église, la théologie et le mouvement altermondialiste.

\section{Abstract}

The altermondialism movement has been hailed by many as promising, and is explicitly based upon the idea of coming together across geographic borders and differences to organize collectively for global justice. In recent years, however, the altermondialsm movement and the World Social Forums (WSFs) have come under some serious criticism and as early as the second WSF in 2002, some were beginning to question if these gatherings were in fact lending themselves to the "the colonization of resistance" (Mngxitama 2005). Others have noticed and commented on the dominance of groups 
from the global North at these events. The objective of this article is to consider the racialized positioning of Church groups and members from the global North. The approach that I use to understand the processes of racialization is largely shaped by feminist, postcolonial and/or antiracist methodologies, which seek to expose the ways knowledge is produced to create and sustain unequal relations and to constitute subjectivities through racialized representations and modes of thinking. One of the practical questions that this study raises is whether activists from the Global North should be participating in such movements at all. In raising such questions, the article encourages careful contemplation of North/South power relations in global justice movements power and suggest a need for more sustained research and scholarship into the links between, Church, theology, and the altermondialism movement. 